\title{
Cochlear Implantation in Patients with Eosinophilic Otitis Media
}

\author{
Masahiro Takahashi ${ }^{*}$, Yasuhiro Arai1, Naoko Sakuma1, Daisuke Sano1, \\ Goshi Nishimura1, Takahide Taguchi', Nobuhiko Oridate', Satoshi Iwasaki², \\ Shin-Ichi Usami ${ }^{3}$ \\ ${ }^{1}$ Department of Otorhinolaryngology Head and Neck Surgery, Yokohama City University School of Medicine, \\ Yokohama, Japan \\ ${ }^{2}$ Department of Otorhinolaryngology, International University of Health and Welfare, Mita Hospital, Tokyo, \\ Japan \\ ${ }^{3}$ Department of Otorhinolaryngology, Shinshu University School of Medicine, Nagano, Japan \\ Email: ${ }^{*}$ masa12 1@yokohama-cu.ac.jp
}

Received 22 December 2014; accepted 10 January 2015; published 22 January 2015

Copyright (C) 2015 by authors and Scientific Research Publishing Inc.

This work is licensed under the Creative Commons Attribution International License (CC BY).

http://creativecommons.org/licenses/by/4.0/

(c) () Open Access

\section{Abstract}

It is known that cochlear implantation for deaf patients with eosinophilic otitis media (EOM) is safe and can provide good speech perception. However, the best timing of implant surgery in patients with EOM is not yet known. The aim of this case report is to suggest the appropriate timing of the surgery in EOM patients with deaf. Cochlear implantation was indicated in two patients with EOM. One underwent cochlear implantation in the absence of any ear discharge. In the other case, implant surgery was delayed for three years due to persistent ear discharge. No complications related to implant device or skin flap were observed in either case. The speech recognition score after implantation was good in the first case and poor in the second case. Perioperative complications were manageable even in the patient with persistent ear discharge. However, the delay in implant surgery due to the persistent ear discharge resulted in a poor speech recognition score. Early implantation should be considered even in EOM patients with ear discharge, although the presence of active middle ear inflammation is regarded as one of the contraindications for implantation according to the current Japanese guidelines.

\section{Keywords}

Eosinophilic Otitis Media, Cochlear Implantation, Speech Recognition

${ }^{*}$ Corresponding author.

How to cite this paper: Takahashi, M., Arai, Y., Sakuma, N., Sano, D., Nishimura, G., Taguchi, T., Oridate, N., Iwasaki, S. and Usami, S.-I. (2015) Cochlear Implantation in Patients with Eosinophilic Otitis Media. International Journal of Otolaryngology and Head \& Neck Surgery, 4, 32-37. http://dx.doi.org/10.4236/ijohns.2015.41006 


\section{Introduction}

Eosinophilic otitis media (EOM) is an intractable middle ear disease with eosinophil-enriched middle ear effusion [1] [2]. The most common characteristic of EOM is the presence of highly viscous mucoid middle ear effusion enriched with eosinophils, although the mechanism of eosinophil accumulation in the middle ear has not yet been determined. The deterioration of bone conductive hearing level (BCHL) is more frequently observed in patients with EOM than that in those with chronic otitis media (COM) [3], with 47\% of EOM patients showing deterioration in BCHL and 6\% developing profound hearing loss [4]. It has been reported that cochlear implantation is safe and provides good speech recognition for EOM patient with deafness [5].

We experienced two cases of EOM in which the patients required cochlear implant. The patients showed different speech recognition scores after implantation, and we herein discuss the reason for this difference.

\section{Case}

Case 1. A 53-year-old female was referred to our hospital for the investigation on progressive bilateral sensorineural hearing loss. Thirty years previously, she had been diagnosed with bilateral chronic otitis media (COM). She was also diagnosed with chronic sinusitis without nasal polyposis, and she had been treated with steroid inhalation therapy for bronchial asthma. At the age of 49, bilateral profound sensorineural hearing loss was detected. On pathological examination, the middle ear mucosa contained numerous eosinophils and she was diagnosed with EOM according to the 2011 diagnostic criteria [6]. Pure tone audiometry showed bilateral profound hearing loss (Figure 1). Her speech recognition score was 10\% (Japanese monosyllable list) in both ears. Temporal bone computed tomography (CT) and magnetic resonance imaging (MRI) showed no low density tissue in mastoid or tympanic cavities (Figure 2(a)). No ossification of the cochlea was evident (Figure 2(b)). As she did not present with any ear discharge, we recommend cochlear implantation. After systemic administration of a corticosteroid (dexamethazon $2 \mathrm{mg}$ /day $\times 7$ days), we performed cochlear implantation through the round window approach with miryngoplasty in her left ear. As no ossification of the cochlea was observed, flex electrode arrays (Med-EL Flex ${ }^{28}$ ) were used and inserted completely. No complications in relation to the implant device or skin flap were observed during a three-year follow-up. In spite of postoperative oral steroid administration, the tympanic membrane was re-perforated and ear discharge recurred at six months post-surgery (Figure 3). Even

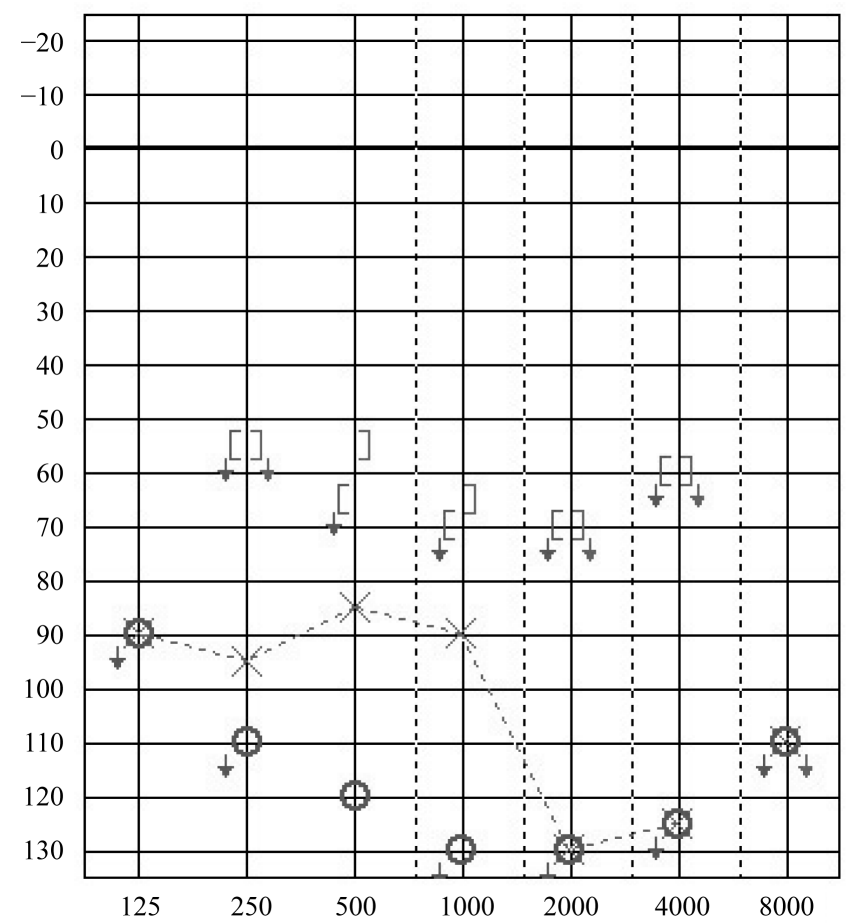

Figure 1. Preoperative pure tone audiometry of Case 1. 


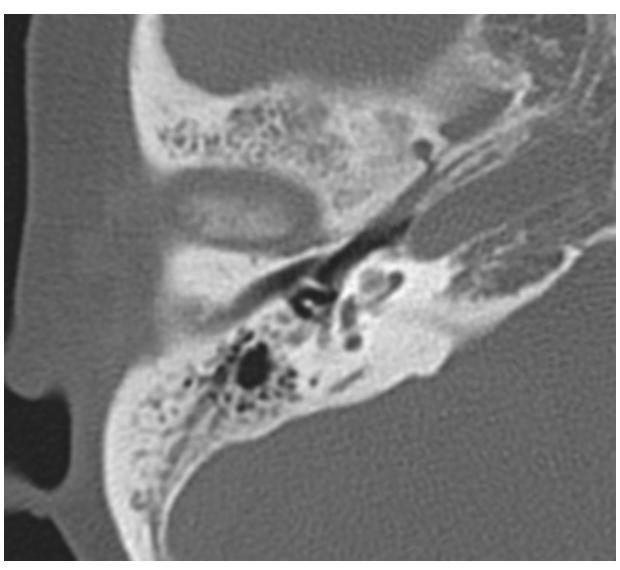

(a)

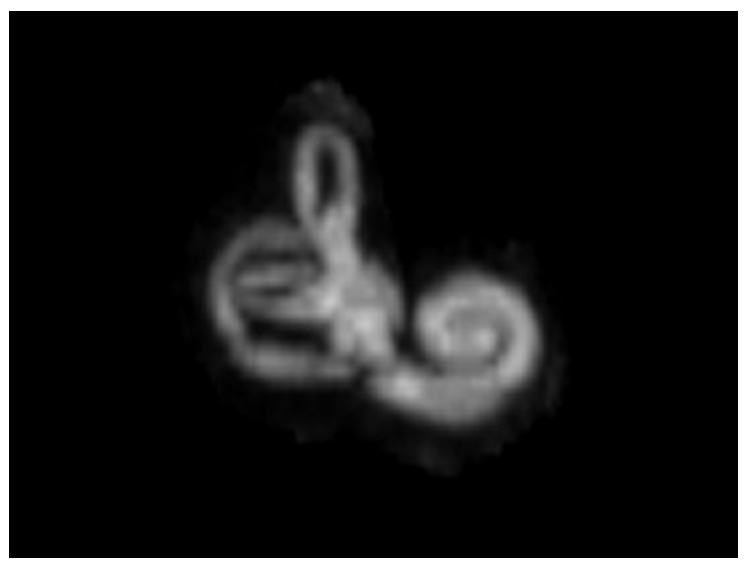

(b)

Figure 2. (a) Preoperative CT of Case 1. No low density tissue in mastoid and tympanic cavity; (b) Preoperative 3D MRI of Case 1. Ossified cochlea was not evident.

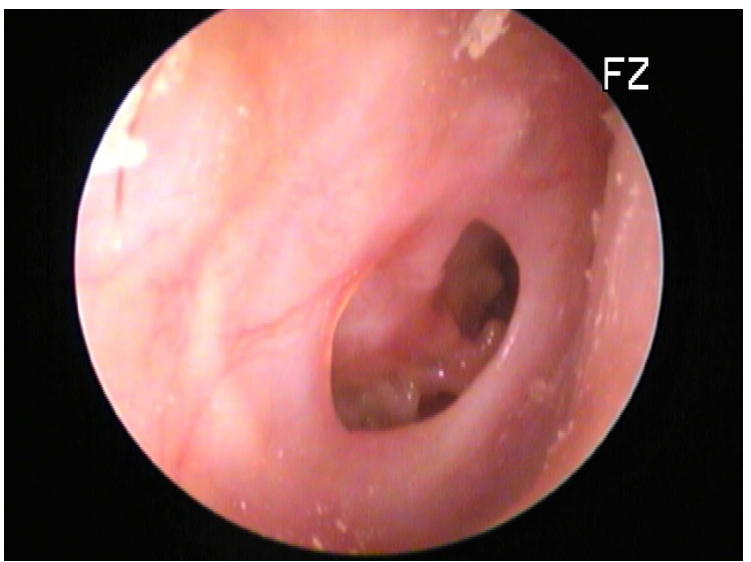

Figure 3. The right tympanic membrane of Case 1 was reperforated and ear discharge was recurred.

under such conditions, her speech recognition score reached $92 \%$ (Japanese monosyllable list) at three years after surgery.

Case 2. A 65-year-old female was referred to our hospital for the investigation on progressive bilateral sensorineural hearing loss. She had been diagnosed with bilateral COM 12 years previously and she had been suffering from ear discharge. She had also been treated with oral steroid therapy for bronchial asthma. She was also diagnosed with chronic sinusitis with nasal polyposis. The middle ear viscous discharge contained numerous eosinophils and she was diagnosed with EOM according to the 2011 diagnostic criteria [6]. Temporal bone CT showed low density areas in the mastoid and tympanic cavities but MRI showed no ossification of the cochlea (Figure 4(a), Figure 4(b)). In spite of anti-IgE therapy in combination with oral steroids, an anti-histaminergic agent and leukotriene receptor antagonist administration, her hearing threshold was gradually deteriorated, and she developed bilateral profound hearing loss at the age of 70 (Figure 5). Her speech recognition score was 20\% (Japanese monosyllable list) in both ears. As the middle ear discharge was persistent, we decided not to perform the cochlear implant at that time. 3 years later, she insisted on receiving a cochlear implant since she could not hear at all with hearing aids. The electrode array (Med-EL Flex ${ }^{28}$ ) was inserted into the scala vestibule in her left ear as ossification of the scala tympani was found during the surgery. Myringoplasty was also performed in her left ear. No complications in relation to the implant device or skin flap were observed during a two-year followup. In spite of postoperative oral steroid administration, the tympanic membrane was re-perforated and ear discharge recurred three months post-surgery (Figure 6). Her speech recognition score was 20\% (Japanese monosyllable list). 


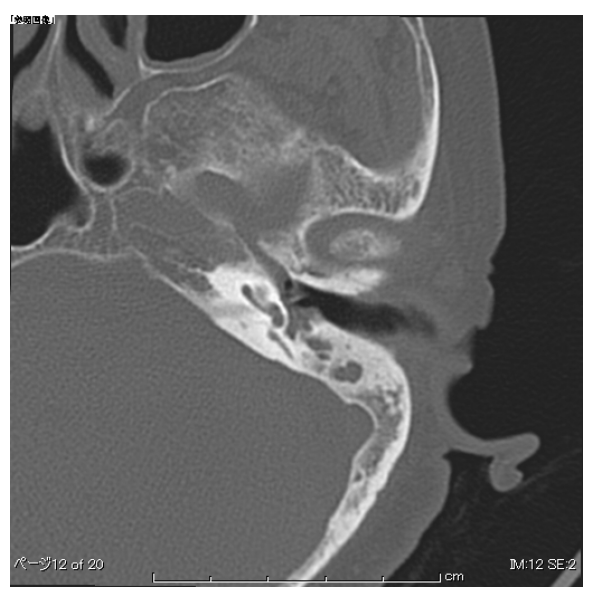

(a)

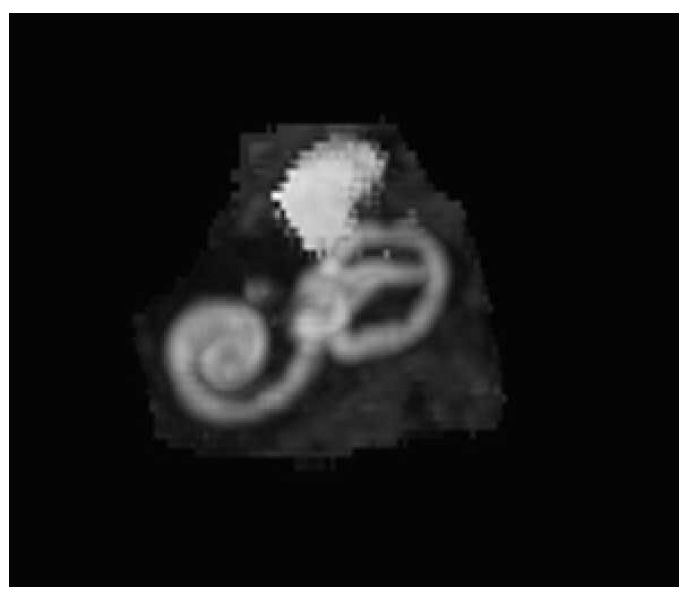

(b)

Figure 4. (a) Preoperative CT of Case 2. Low density area in mastoid and tympanic cavity; (b) Preoperative 3D MRI of Case 2. Ossified cochlea was not evident.

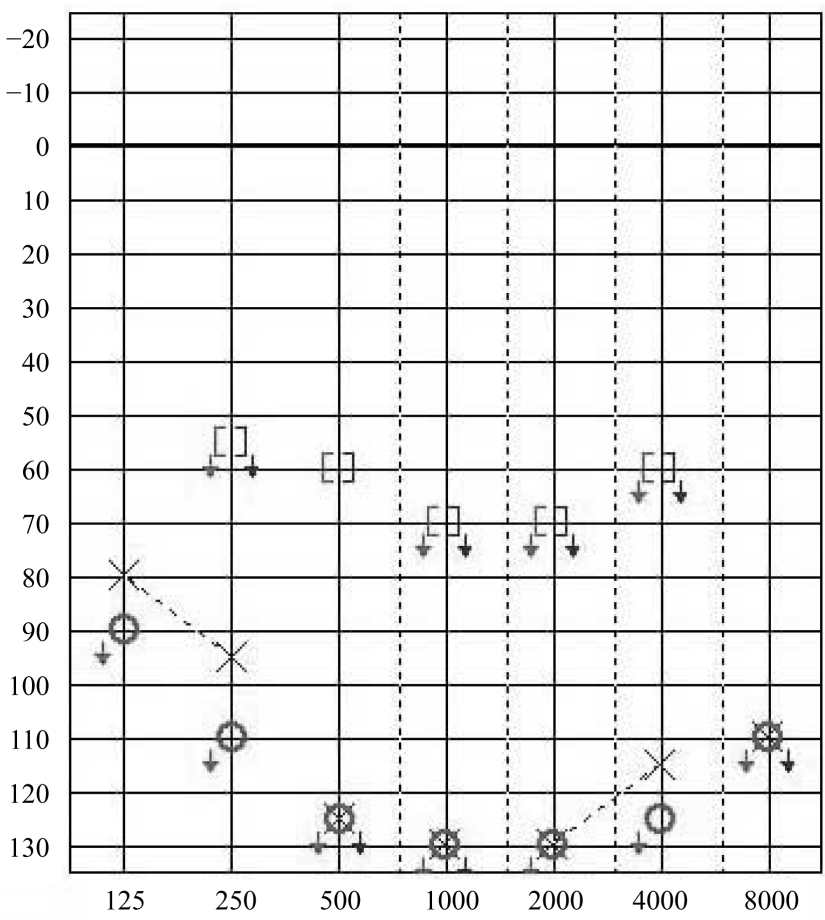

Figure 5. Preoperative pure tone audiometry of Case 2.

\section{Discussion}

EOM causes marked damage to sensorineural hearing, with the rate of BCHL loss being about ten times greater than that for chronic suppurative otitis media [3]. High-tone hearing loss, in particular, is more frequent and more severe in EOM patients than in COM patients [7], with 47\% of EOM patients showing deterioration in BCHL, and 6\% developing bilateral profound hearing loss according to the clinical survey of EOM in Japan [4]. The cause of deterioration in BCHL in cases of EOM remains unclear, but significantly higher concentrations of eosinophil cationic protein (ECP) and IgE have been detected in the middle ear effusion of EOM patients than in that of control patients [8]. Further, numerous eosinophil infiltrations were in the scala tympani, as well as severe morphological damage to the cochlea, the organ of Corti and the stria vascularis was observed in the prolonged topical stimulation animal model and is thought to be the cause of the deterioration in BCHL [9]. 


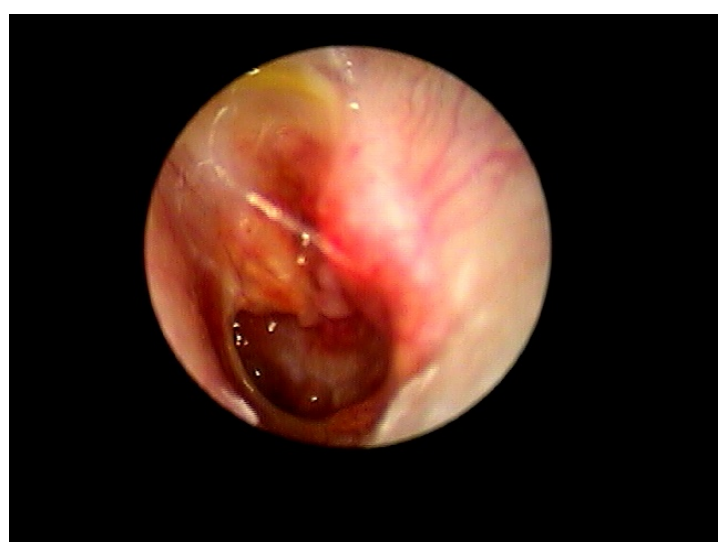

Figure 6. The left tympanic membrane of Case 2 was re-perforated and ear discharge was recurred.

Patients with EOM and profound sensorineural hearing loss are considered to be good candidates for cochlear implantation, and the results of speech recognition testing after implantation have shown marked improvements [5].

However, the speech recognition scores after implantation were quite different between the two cases we experienced. We selected a single-staged cochlear implant in these cases, because a planned-staged (such as mastoidectomy and tympanoplasty) cochlear implant can lead to cochlear ossification [10] and subsequently, to poor results in terms of speech perception after implantation. For cochlear implantation in patients with COM, obliteration and isolation of the middle ear cavity by closure of the external ear canal is required to control the ear discharge [11]. The characteristic of ear discharge of EOM is completely different from that of COM. Appropriate management of EOM for cochlear implant surgery is not yet determined, but the most important event in the perioperative period is thought to be the management of ear discharge.

As we believe that it is difficult to manage the clinical condition of EOM itself, one of options involving closure of the tympanic membrane and external auditory canal at the time of implant surgery was not adopted. Instead, by adding myringoplasty to cochlear implantation, we managed to control short-term postoperative ear discharge. However, in both patients, the tympanic membrane was eventually re-perforated and ear discharge recurred despite postoperative oral steroid administration.

On the other hand, the speech recognition score after implantation differed markedly between the two cases, suggesting that postoperative ear discharge may not necessarily be associated with poor functional outcomes. In such circumstances, and in the light of the fact that no complications were observed during the perioperative period, early implantation could be taken into consideration in cases of deaf patients with EOM showing persistent ear discharge. Further accumulation of cases, however, is still needed to confirm the best timing and method of cochlear implantation in EOM patients with ear discharge.

The long-term results of cochlear implantation in EOM patients were another important issue, especially in patients with persistent ear discharge. It is difficult to manage the clinical condition of EOM, and long-term inflammation within the cochlea affects the cochlear structures, with organs including the organ of Corti, stria vascularis, and spiral ganglion showing signs of degeneration [12]. Although it has been reported that good speech recognition scores after implantation for patients with EOM were maintained for eight years [5], longterm observation is definitely needed to verify the benefits of cochlear implantation in EOM patients.

\section{Conclusion}

In conclusion, in cases where cochlear implantation is indicated for EOM patients, early implantation should be considered, even in patients with persistent ear discharge, as the perioperative complications are manageable and a delayed in cochlear implantation surgery due to the ear discharge may result in cochlear ossification and subsequent poor speech recognition scores.

\section{Conflict of Interest}

The authors declare that there is no conflict of interest. 


\section{References}

[1] Tomioka, S., Kobayashi, T. and Takasaka, T. (1997) Intractable Otitis Media in Patients with Bronchial Asthma (Eosinophilic Ottis Media). In: Sanna, M., Ed., Cholesteatoma and Mastoid Surgery, CICI Edizioni International, Rome, 851-853.

[2] Nagamine, H., Iino, Y., Kojima, C., Miyazawa, T. and Iida, T. (2002) Clinical Characteristics of So-Called Eosinophilic Otitis Media. Auris Nasus Larynx, 29, 19-28. http://dx.doi.org/10.1016/S0385-8146(01)00124-9

[3] Nakagawa, T., Matubara, A., Shiratsuchi, H., Kakazu, Y., Nakashima, T., Koike, K., Umezaki, T. and Komune, S. (2006) Intractable Otitis Media with Eosinophils: Importance of Diagnosis and Validity of Treatment for Hearing Preservation. ORL, 68, 118-122. http://dx.doi.org/10.1159/000091215

[4] Suzuki, H., Matsutani, S. and Kawase, T. (2004) Epidemiologic Surveillance of “Eosinophilic Otitis Media” in Japan. Otology Japan, 14, 112-117. (In Japanese)

[5] Iwasaki, S., Nagura, M. and Mizuta, K. (2006) Cochlear Implantation in a Patient with Eosinophilic Otitis Media. European Archives of Oto-Rhino-Laryngology, 263, 365-369. http://dx.doi.org/10.1007/s00405-005-1006-2

[6] Iino, Y., Matsutani, S., Matsubara, A., Nakagawa, T. and Nonaka, M. (2011) Diagnostic Criteria of Eosinophilic Otitis Media, a Newly Recognized Middle Ear Disease. Auris Nasus Larynx, 38, 456-461. http://dx.doi.org/10.1016/j.anl.2010.11.016

[7] Iino, Y., Usubuchi, H., Kodama, K., Takizawa, K., Kanazawa, T. and Ohta, Y. (2008) Bone Conduction Hearing Level in Patients with Eosinophilic Otitis Media Associated with Bronchial Asthma. Otology Neurotology, 29, 949-952. http://dx.doi.org/10.1097/MAO.0b013e318185fb0d

[8] Iino, Y., Usubuchi, H., Kodama, K., Kanazawa, H., Takizawa, K., Kanazawa, T. and Ohta, Y. (2010) Eoshinophilic Inflammation in the Middle Ear Induces Deterioration of Bone-Conduction Hearing Level in Patients with Eosinophilic Otitis Media. Otology Neurotology, 31, 100-104. http://dx.doi.org/10.1097/MAO.0b013e3181bc3781

[9] Matubara, A., Nishizawa, H., Kurose, A., Nakagawa, T., Takahata, J. and Sasaki, A. (2014) An Experimental Study of Inner Ear Injury in an Animal Model of Eosinophilic Otitis Media. Acta Oto-Laryngologica, 134, 227-32. http://dx.doi.org/10.3109/00016489.2013.859395

[10] Himi, T., Harabuchi, Y., Shintani, T., Yamaguchi, T., Yoshida, I. and Kataura, A. (1997) Surgical Strategy of Cochlear Implantation in Patients with Chronic Middle Ear Disease. Audiology and Neurotology, 2, 410-417. http://dx.doi.org/10.1159/000259266

[11] Kojima, H., Sakurai, Y., Rikitake, M., Tanaka, Y., Kawano, A. and Moriyama, H. (2010) Cochlear Implantation in Patients with Chronic Otitis Media. Auris Nasus Larynx, 37, 415-421. http://dx.doi.org/10.1016/j.anl.2010.01.009

[12] Keithley, E. and Haris, J. (1996) Late Sequelae of Cochlear Infection. Laryngoscope, 106, 341-345. http://dx.doi.org/10.1097/00005537-199603000-00019 
Scientific Research Publishing (SCIRP) is one of the largest Open Access journal publishers. It is currently publishing more than 200 open access, online, peer-reviewed journals covering a wide range of academic disciplines. SCIRP serves the worldwide academic communities and contributes to the progress and application of science with its publication.

Other selected journals from SCIRP are listed as below. Submit your manuscript to us via either submit@scirp.org or Online Submission Portal.
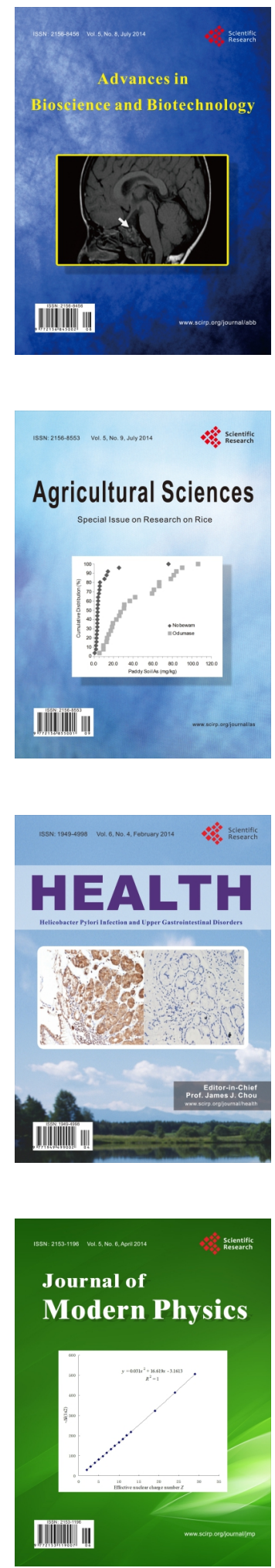
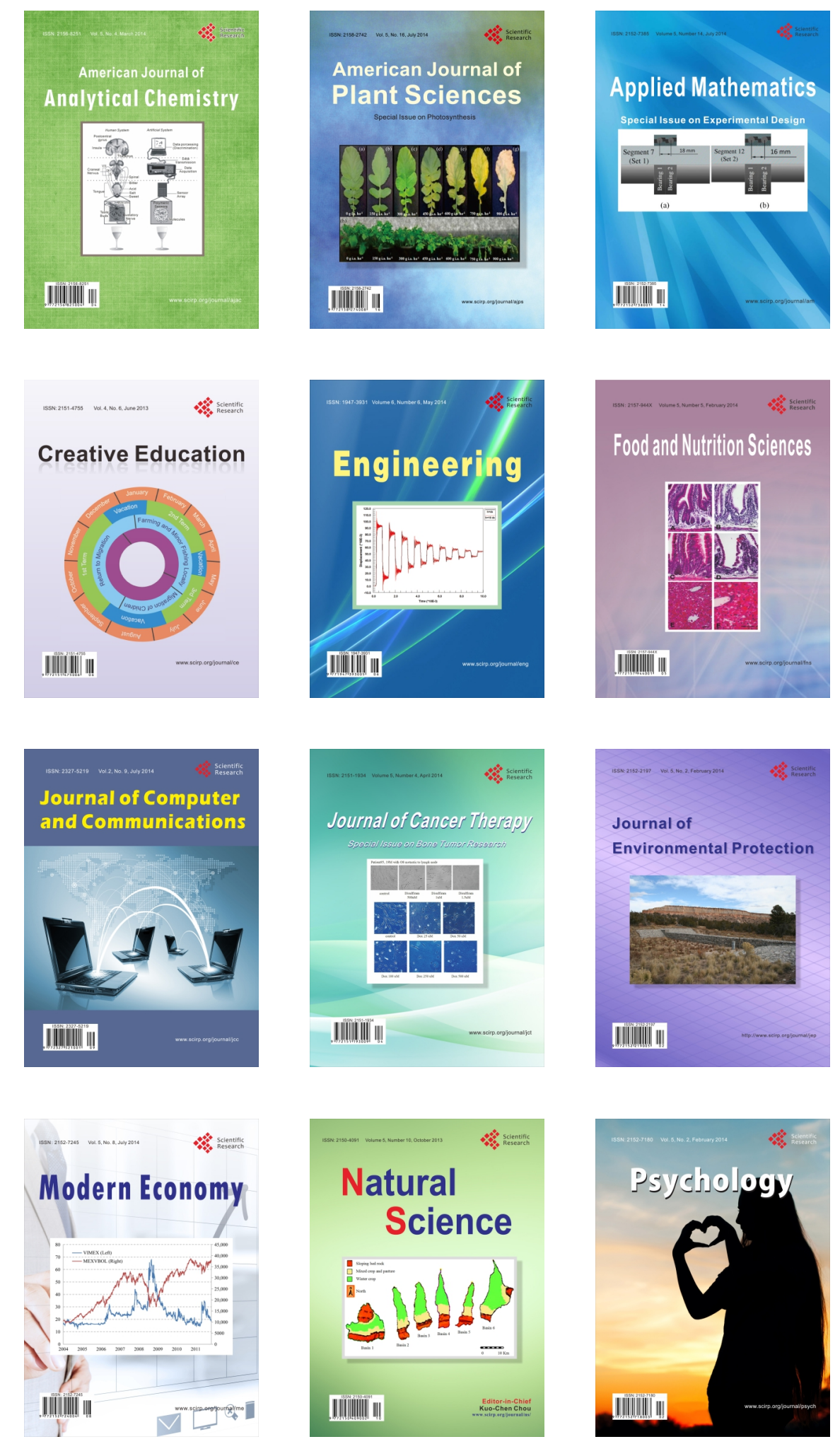